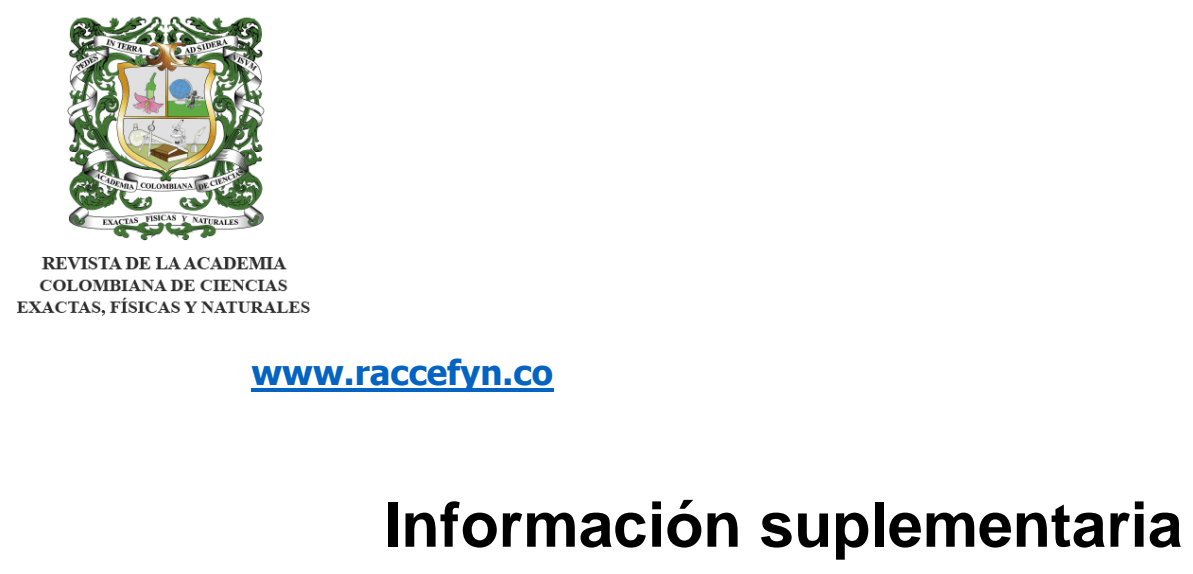

Estimación espaciotemporal de la recarga potencial en un sistema pseudokárstico tropical

Spatio-temporal estimation of the potential recharge in a tropical pseudokarst system

Sandra M. Patiño-Rojas, Marcela Jaramillo *

majaram9@eafit.edu.co

\title{
Contenido
}

- Figuras suplementarias S1 a S5

- Tablas suplementarias S1 a S6 


\section{Figuras suplementarias}

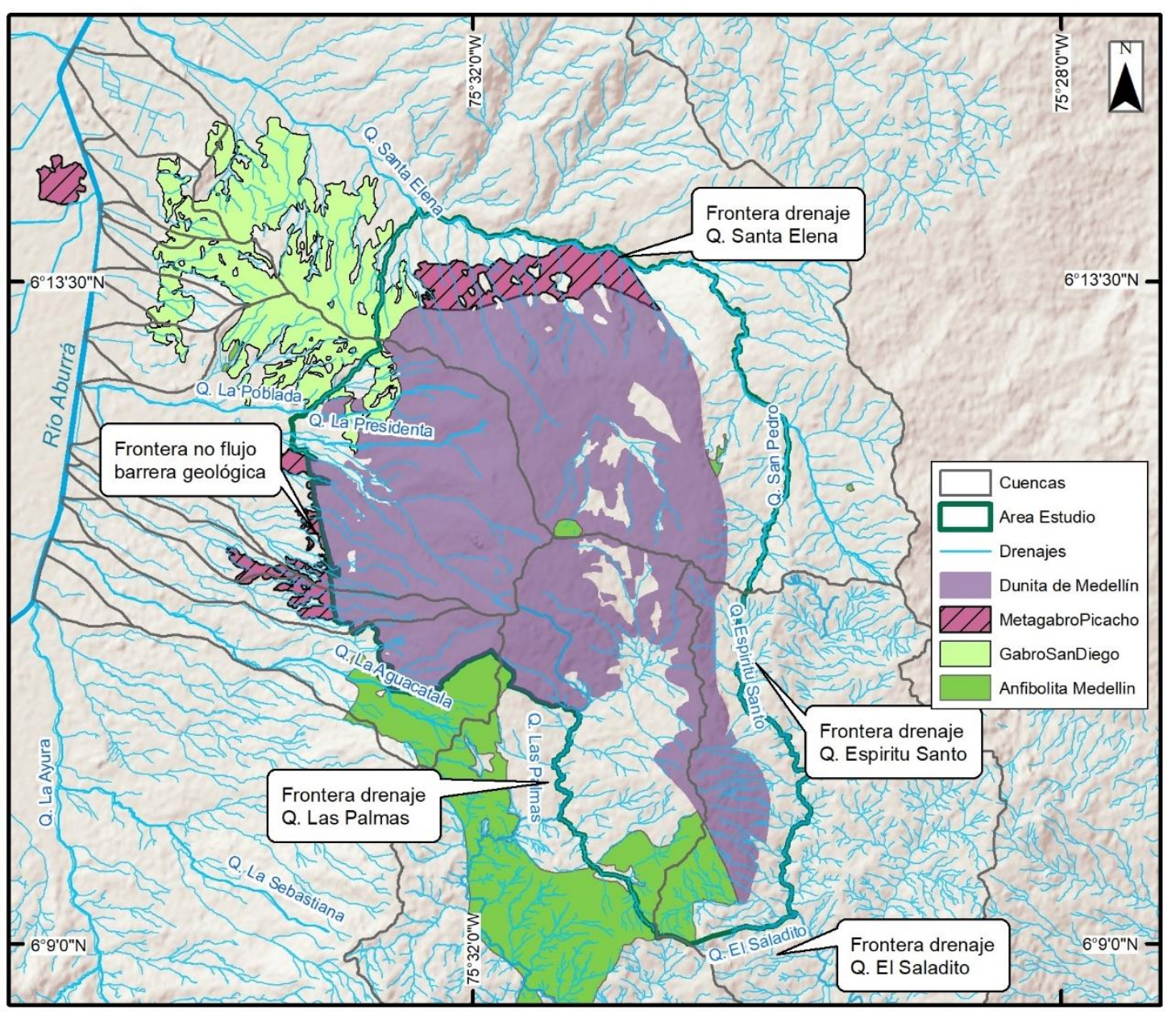

Figura S1. Fronteras del dominio de modelación. Fuente: Elaboración propia. 


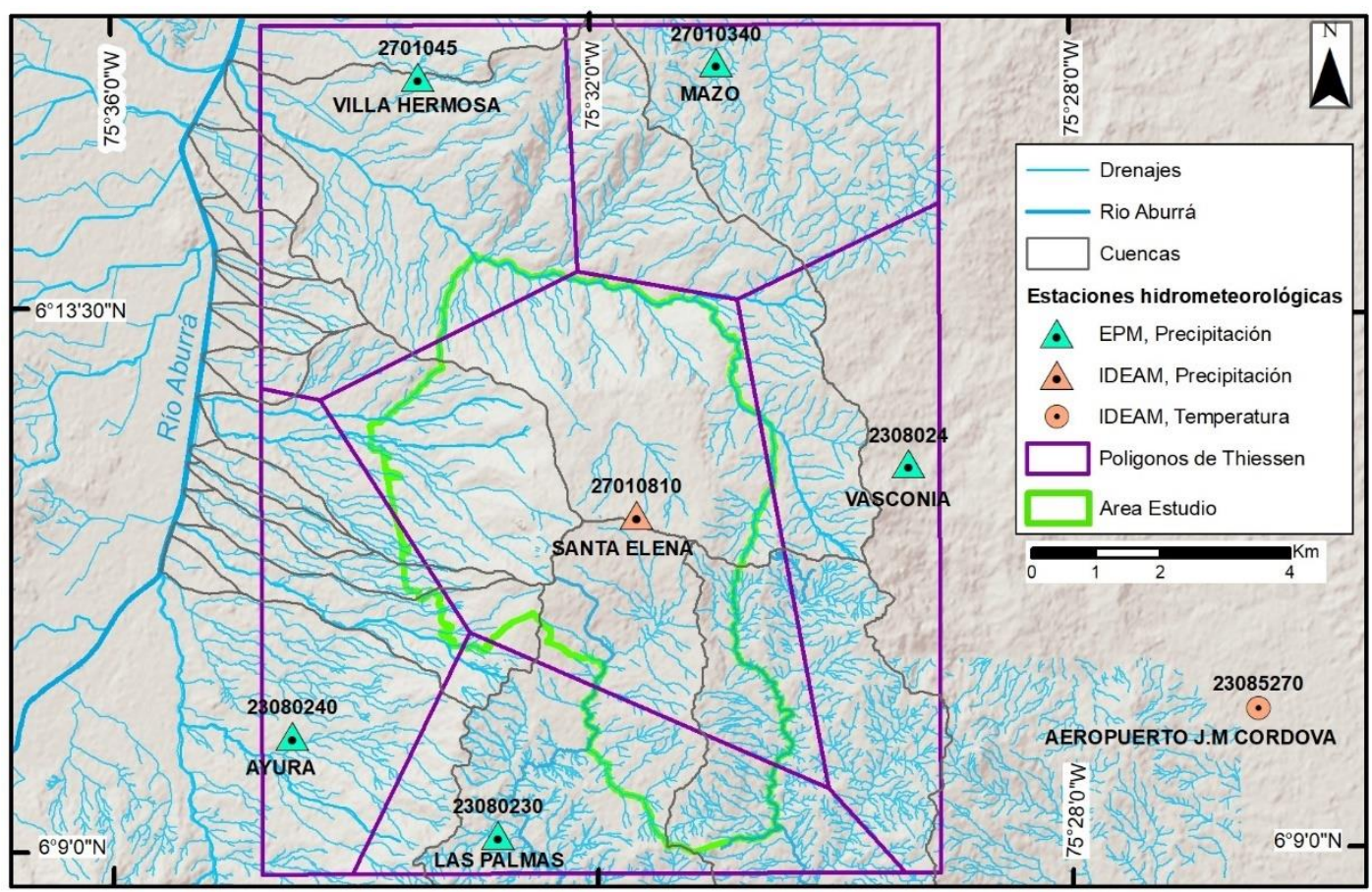

Figura S2. Distribución espacial de las estaciones de precipitación y temperatura disponibles. Fuente: Elaboración propia. 


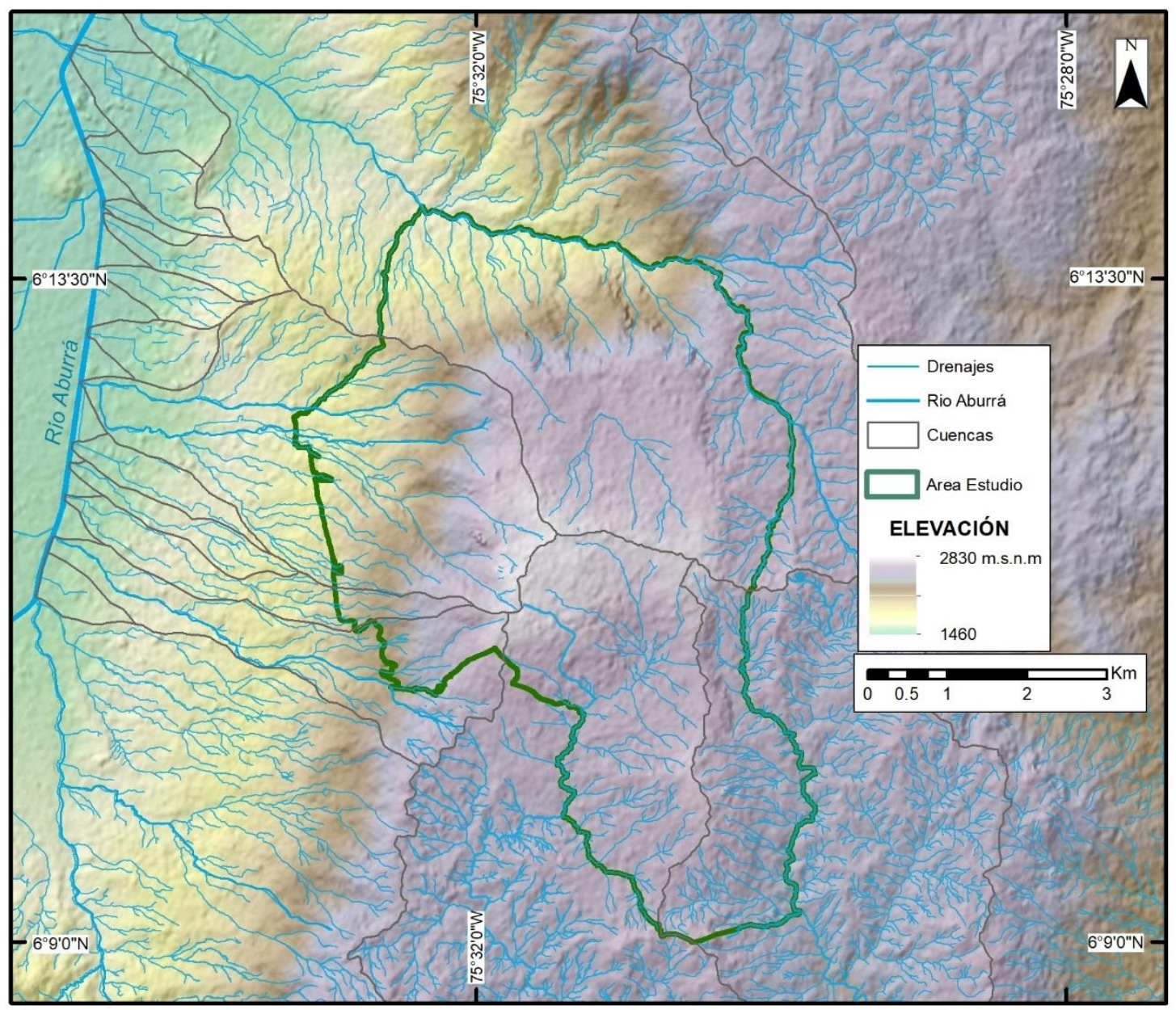

Figura S3. DEM $12.5 \mathrm{~m}$ x12.5 m empleado en el estudio y red de drenajes del proyecto SIGMA. Fuente: Elaboración propia. 


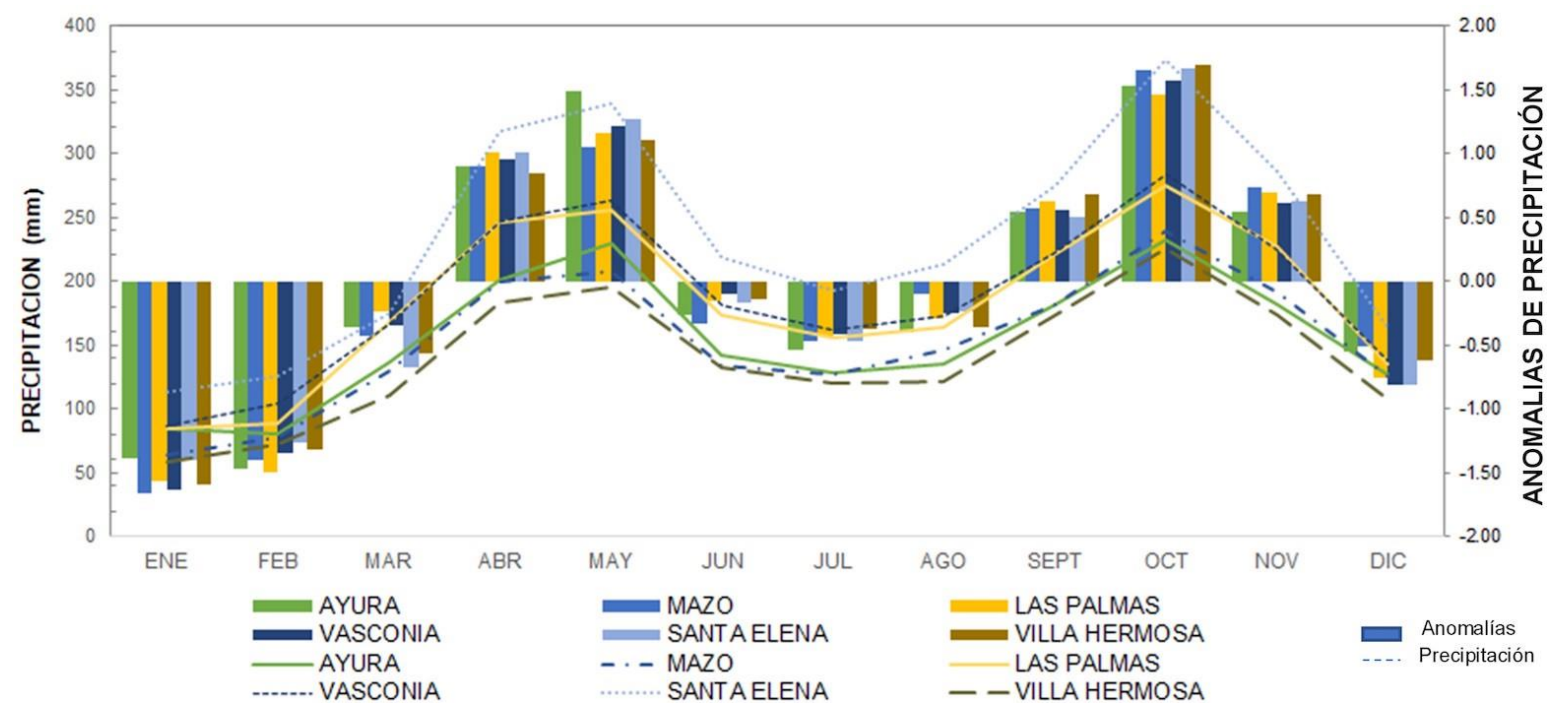

Figura S4. Ciclo promedio multianual y anomalías de precipitación para las estaciones en la zona de estudio. Elaboración propia. 


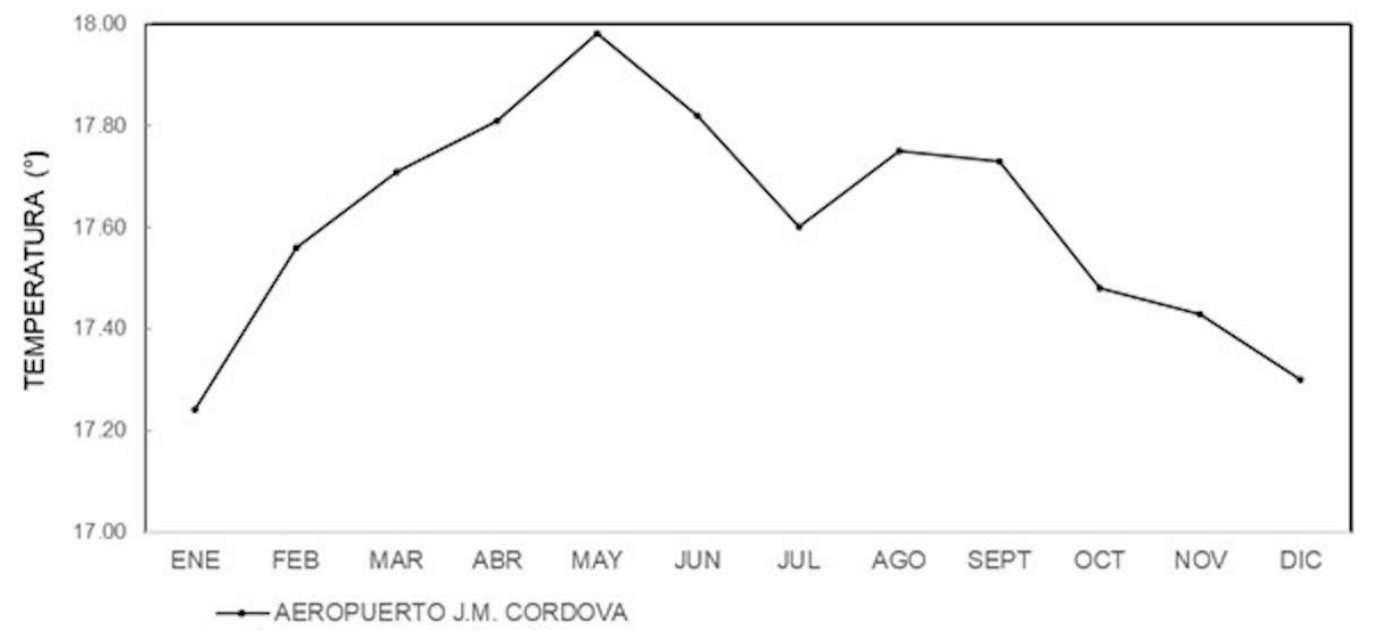

Figura S5. Ciclo promedio multianual de temperatura. Elaboración propia. 


\section{Tablas suplementarias}

Tabla S1. Estaciones hidrometeorológicas con información empleada en la determinación de la recarga.

\begin{tabular}{cclcccccc}
\hline Fuente & \multirow{2}{*}{ Código } & \multicolumn{1}{c}{ Estación } & Tipo & $\begin{array}{c}\text { Coordenadas } \\
\text { Lat. }\end{array}$ & $\begin{array}{c}\text { Elevación } \\
\text { Long.s.n.m.) }\end{array}$ & $\begin{array}{c}\text { Periodo de } \\
\text { registro }\end{array}$ & $\begin{array}{c}\% \text { datos } \\
\text { faltantes }\end{array}$ \\
\hline EPM & 27010930 & AYURÁ & $P G$ & 6.16 & -75.63 & 1770 & $1972-2019$ & 0.43 \\
EPM & 23080230 & LAS PALMAS & PG & 6.15 & -75.53 & 2495 & $1948-2019$ & 0.65 \\
EPM & 27010340 & MAZO & $P G$ & 6.26 & -75.52 & 2455 & $1948-2019$ & 0.71 \\
EPM & 23080240 & VASCONIA & PG & 6.20 & -75.48 & 2510 & $1948-2019$ & 0.53 \\
EPM & 2701045 & VILLA HERMOSA & PG & 6.24 & -75.55 & 1680 & $1948-2019$ & 0.43 \\
IDEAM & 23085270 & AEROPUERTO J.M. CORDOVA & SP & 6.17 & -75.43 & 2157 & $1978-2019$ & 0.00 \\
IDEAM & 27010810 & SANTA ELENA & CO & 6.20 & -75.52 & 2250 & $1971-2019$ & 1.22 \\
\hline
\end{tabular}


Tabla S2. Descripción de las coberturas del área en estudio definidas según la metodología Corine Land Cover adaptada para Colombia.

\begin{tabular}{|c|c|c|c|c|}
\hline Código & Descripción & $\begin{array}{c}\text { Tipo de } \\
\text { cobertura }\end{array}$ & $\begin{array}{l}\text { Área } \\
\text { (ha) }\end{array}$ & $\begin{array}{c}\% \text { que ocupa en } \\
\text { la zona }\end{array}$ \\
\hline 1.1 .1 & Tejido urbano continuo & \multirow{7}{*}{$\begin{array}{l}\text { Territorios } \\
\text { artificializados }\end{array}$} & 138.2 & 3.9 \\
\hline 1.1 .2 & Tejido urbano discontinuo & & 386.9 & 10.8 \\
\hline 1.1.3 & Tejido urbano discontinuo & & 90.6 & 2.5 \\
\hline 1.2 .1 & Zonas industrias y comerciales & & 4.3 & 0.1 \\
\hline 1.2 .2 & Red vial, ferroviaria y terrenos asociados & & 6.6 & 0.2 \\
\hline 1.4 .1 & Zonas verdes urbanas & & 77.8 & 2.2 \\
\hline 1.4 .2 & Instalaciones recreativas & & 5.2 & 0.1 \\
\hline 2.1 .1 & Otros cultivos transitorios & \multirow{14}{*}{$\begin{array}{l}\text { Territorios } \\
\text { agrícolas }\end{array}$} & 16.3 & 0.5 \\
\hline 2.1 .2 & Cereales & & 6.3 & 0.2 \\
\hline 2.1 .3 & Oleaginosas y leguminosas & & 3.1 & 0.1 \\
\hline 2.2 .1 & Cultivos permanentes herbáceos & & 6.2 & 0.2 \\
\hline 2.2 .2 & Cultivos permanentes arbustivos & & 50.4 & 1.4 \\
\hline 2.2 .5 & Cultivos confinados & & 2.9 & 0.1 \\
\hline 2.3 .1 & Pastos limpios & & 875.9 & 24.5 \\
\hline 2.3 .2 & Pastos arbolados & & 6.2 & 0.2 \\
\hline 2.3 .3 & Pastos enmalezados & & 97.0 & 2.7 \\
\hline 2.4 .1 & Mosaico de cultivos & & 32.6 & 0.9 \\
\hline 2.4 .2 & Mosaico de pastos y cultivos & & 37.9 & 1.1 \\
\hline 2.4 .3 & $\begin{array}{l}\text { Mosaico de cultivos, pastos y espacios } \\
\text { naturales }\end{array}$ & & 4.3 & 0.1 \\
\hline 2.4 .4 & $\begin{array}{l}\text { Mosaico de pastos con espacios } \\
\text { naturales }\end{array}$ & & 2.9 & 0.1 \\
\hline 2.4 .5 & $\begin{array}{l}\text { Mosaico de cultivos con espacios } \\
\text { naturales }\end{array}$ & & 4.2 & 0.1 \\
\hline 3.1 .1 & Bosque denso & \multirow{8}{*}{$\begin{array}{l}\text { Bosques y } \\
\text { área } \\
\text { seminaturales }\end{array}$} & 0.3 & 0.0 \\
\hline 3.1 .2 & Bosque abierto & & 41.7 & 1.2 \\
\hline 3.1 .3 & Bosque fragmentado & & 749.1 & 21.0 \\
\hline 3.1 .4 & Bosque de galería y ribereño & & 204.7 & 5.7 \\
\hline 3.1 .5 & Plantación forestal & & 27.1 & 0.8 \\
\hline 3.2 .2 & Arbustal & & 5.1 & 0.1 \\
\hline 3.2 .3 & Vegetación secundaria o transición & & 676.4 & 18.9 \\
\hline 3.3 .3 & Tierras desnudas y degradadas & & 10.9 & 0.3 \\
\hline
\end{tabular}


Tabla S3. Profundidad de las raíces $(\mathrm{cm})$ para las combinaciones Textura-

Cobertura

\begin{tabular}{|c|c|c|c|c|c|c|c|c|c|c|c|c|c|c|c|c|}
\hline \multirow{2}{*}{ Suelo } & \multirow{2}{*}{ Textura } & \multicolumn{15}{|c|}{ Cobertura nivel 3} \\
\hline & & 2.4 .2 & 2.4 .3 & 2.2.2 & 1.1 .1 & 1.1.2 & 1.4.1 & 3.2.3 & 1.2 .1 & 2.3.1 & 2.4 .1 & 1.1 .3 & 3.1 .4 & 3.2.3 & 3.1 .5 & 2.3.3 \\
\hline Alc-1 & Limoarenosa & 172.8 & 173.7 & 158.4 & & & & & & & & & & & & \\
\hline ME-a2 & Arenolimosa & & & & 161.5 & 161.5 & & & & & & & & & & \\
\hline ME_fv2 & Limosa & & & & & & 100.5 & & & & & & & & & \\
\hline ME-fv4 & Arenolimosa & & & & & & & 100.5 & & & & & & & & \\
\hline MS-fv2 & Arenolimosa & & & 158.5 & 161.5 & 161.5 & & 100.5 & 76.2 & 15.2 & 161.5 & & & & & 15.2 \\
\hline Ms_fv1 & Arcillolimosa & & & & 106.7 & & & & & & & & & & & \\
\hline LC & Arcillolimosa & & & & & & & & & 15.2 & 76.2 & 76.2 & 15.2 & & & \\
\hline SD & Arcillolimosa & & & & & & & & & & & 76.2 & 100.5 & & & \\
\hline TE & Limosa & & & & & & & 100.6 & & & & & & 100.5 & 100.5 & 15.2 \\
\hline
\end{tabular}


Tabla S4. Configuración general del modelo SWB

\begin{tabular}{|c|c|}
\hline Sistema de coordenadas & WGS84 \\
\hline \multirow[t]{4}{*}{ Dominio del modelo } & W: -75.554 \\
\hline & $\mathrm{N}: 6.149$ \\
\hline & W: -75.495 \\
\hline & $\mathrm{N}: 6.233$ \\
\hline \multirow[t]{3}{*}{ Dimensiones } & Número de filas: 923 \\
\hline & Número de columnas: 651 \\
\hline & Tamaño de celda: $10 \mathrm{~m}$ \\
\hline Paso de tiempo & Diario \\
\hline $\begin{array}{l}\text { Precipitación puntual o } \\
\text { distribuida }\end{array}$ & Puntual (Estación Santa Elena) \\
\hline $\begin{array}{l}\text { Temperatura puntual o } \\
\text { distribuida }\end{array}$ & Puntual (Estación Aeropuerto J.M. Córdova) \\
\hline Temporada húmeda & Bimodal: Abril - Mayo; Octubre - Noviembre \\
\hline $\begin{array}{l}\text { Parámetro de abstracción } \\
\text { inicial }^{\star}\end{array}$ & 0.05 \\
\hline Método de evapotranspiración & $\begin{array}{l}\text { Thornthwaite-Mather (1957) Estimada a partir de datos } \\
\text { de temperatura puntuales de la estación } \\
\text { AEROPUERTO J.M. CORDOVA }\end{array}$ \\
\hline $\begin{array}{l}\text { Capacidad de almacenamiento } \\
\text { en el suelo }\end{array}$ & $\begin{array}{l}\text { Profundidad de las raíces, calculada según Bradbury et } \\
\text { al., } 2000\end{array}$ \\
\hline Humedad inicial en el suelo & $100 \%$ de capacidad de campo \\
\hline Periodo de simulación & Enero 2009 - diciembre 2017 \\
\hline Años de análisis & $\begin{array}{l}\text { Año Húmedo (Nov 2010-Oct 2011) } \\
\text { Año Seco (Nov 2015-Oct 2016) } \\
\text { Año Normal (Nov 2013-Oct 2014) }\end{array}$ \\
\hline Escala de salidas & Mensual y anual \\
\hline Variables de salida & $\begin{array}{l}\text { Recarga potencial directa, evapotranspiración real, } \\
\text { escorrentía directa }\end{array}$ \\
\hline $\begin{array}{l}\text { *Eliminación de agua de cualqui } \\
\text { temporal. Es definido por el usu } \\
\text { simulaciones diarias y continuas } \\
\text { factor de } 0.05 \text {. }\end{array}$ & $\begin{array}{l}\text { er fuente, ya sea permanentemente o de forma } \\
\text { ario y típicamente toma valores de } 0.2 \text {, aunque para } \\
\text { Woodward et al. (2003) recomiendan el uso de un }\end{array}$ \\
\hline
\end{tabular}


Tabla S5. Configuración general del modelo Schosinsky

\begin{tabular}{ll}
\hline Variables de entrada & Precipitación mensual (mm) \\
& Evapotranspiración potencial Thornthwaite-Mather (1957) \\
& (mm/mes) \\
& Permeabilidad de la capa superficial del suelo $(f c)$ en mm/día \\
& según la clasificación de Schosinsky \& Losilla (2000). \\
& Fracción de lluvia que infiltra por efecto de la pendiente $(K p)$ \\
& según la clasificación de Schosinsky \& Losilla (2000). \\
& Fracción de lluvia que infiltra por efecto de la cobertura (Kv) \\
& según la clasificación de Schosinsky \& Losilla (2000). \\
& Capacidad de campo (CC) y punto de marchitez (PMP) de la \\
& zona homogénea (\%). \\
& Profundidad de las raíces (PR) presentes en la zona \\
& homogénea (mm). \\
& Coeficiente de retención en el follaje (Cfo), que para esta \\
& zona predomina Cfo $=0.12$ porque no hay predominio de \\
& zonas de bosques densos. \\
\hline Años de análisis & Año Húmedo (Nov 2010-Oct 2011) \\
& Año Seco (Nov 2015-Oct 2016) \\
& Año Normal (Nov 2013-Oct 2014) \\
\hline Paso de tiempo & Mensual \\
\hline Precipitación puntual o & Puntual (Estación Santa Elena) \\
distribuida & Calculada por Thornthwaite-Mather (1957) a partir de datos \\
\hline Evapotranspiración & de temperatura de la estación AEROPUERTO J.M. \\
potencial mensual & CORDOVA \\
\hline Variables de salida & Recarga potencial directa, evapotranspiración real, \\
& escorrentía directa \\
\hline
\end{tabular}


Tabla S6. Evapotranspiración potencial (mm/año) para los años de simulación.

\begin{tabular}{llr}
\hline Condición & Periodo & ETP (mm/año) \\
\hline Año normal & $2013-2014$ & 770.2 \\
Año seco (Niño) & $2015-2016$ & 799.9 \\
Año húmedo (Niña) & $2010-2011$ & 756.1 \\
\hline
\end{tabular}

